\title{
Le contrôle organisationnel du risque en contexte hospitalier : un nouveau regard sur le modèle des leviers de contrôle
}

Didier Van Caillie, Professeur Ordinaire, Centre d'Etude de la Performance des Entreprises, HEC Université de Liège, d.vancaillie@ulg.ac.be

Yuliya Shutyak, Chercheuse affiliée au Centre d'Etude de la Performance des Entreprises, HEC Université de Liège ${ }^{1}$

\section{Mots-clés}

Gestion du risque hospitalier - Contrôle de gestion organisationnel - Leviers de contrôle Système de contrôle - Contrôle interactif

\section{Résumé}

L'institution hospitalière est aujourd'hui considérée comme toute autre organisation (WHO, 2003), à savoir un portefeuille de ressources rares (humaines, techniques, immatérielles, financières) qu'il importe de coordonner de manière efficiente (dans une logique de maximisation « outputs/inputs ») en tenant compte explicitement de l'ensemble des risques internes (organisationnels et opérationnels) et externes (risques réglementaires, économiques, sociétaux, technologiques) auxquels elle est confrontée au quotidien (Milgrom, Roberts, 1992). L'atteinte récurrente de cet objectif implique au plan managérial la mise en place d'un véritable système intégré de contrôle de gestion organisationnelle du risque apte à garantir la sécurité économique de l'hôpital en tant qu'organisation à finalité sociétale, système notamment basé sur le modèle des «Leviers de Contrôle» de Simons (1995, p. 180),

\footnotetext{
${ }^{1}$ Cette recherche a fait l'objet d'un financement Prisme par HEC Ecole de Gestion de l'Université de Liège.
} 
particulièrement adapté à la nature des risques médicaux, techniques et sociétaux et aux spécificités des ressources présentes en contexte hospitalier.

\section{Introduction}

Dans la foulée des recommandations formulées par l'Organisation Mondiale de la Santé (WHO, 2003), la manière dont l'institution hospitalière a été considérée par la littérature scientifique en sciences hospitalières et en sciences de gestion a considérablement évolué au cours des 20 dernières années, : l'hôpital est aujourd'hui considéré comme toute autre organisation, à savoir un portefeuille de ressources rares (humaines, techniques, immatérielles, financières) qu'il importe de coordonner de manière efficiente (dans une logique de maximisation « outputs/inputs ») en tenant compte explicitement de l'ensemble des risques internes (organisationnels et opérationnels) et externes (risques réglementaires, économiques, sociétaux, technologiques) auxquels il est confronté au quotidien (Milgrom, Roberts, 1992). L'atteinte récurrente de cet objectif implique dès lors la mise en place d'un véritable système intégré de contrôle de gestion organisationnel apte à garantir la sécurité économique de l'hôpital en tant qu'organisation à finalité sociétale, système notamment basé sur le modèle des «Leviers de Contrôle » de Simons (1995, p. 180), particulièrement adapté à la nature des risques (notamment économiques) et aux spécificités des ressources présentes en contexte hospitalier.

\section{L'institution hospitalière, acteur économique et social et objet de risque}

En 1957 déjà, l'Organisation Mondiale de la Santé a défini l'hôpital comme une partie intégrante d'une organisation sociale et médicale à l'échelle d'un territoire, dont la fonction 
principale est de fournir à sa population une offre complète de soins de santé, tant curatifs que préventifs (WHO, 1963:9).

De ce fait, une institution hospitalière s'intègre inévitablement au sein d'un réseau de multiples parties prenantes, dont elle se doit de rencontrer de manière récurrente les attentes et les exigences dans une double logique d'efficacité et d'efficience : selon ce paradigme, l'efficacité de son action doit être recherchée en veillant, dans une vision organisationnelle basée sur la gestion continue de la rareté des ressources, à maximiser en continu le rapport « outputs/inputs », à savoir en l'occurrence le rapport entre les impacts sur la santé du patient et le volume des ressources rares consommées, tout en maîtrisant le niveau de risque technique (en l'occurrence sanitaire) et économique (le plus souvent budgétaire ou financier) auquel l'institution hospitalière est confrontée.

Comme toute autre organisation, l'hôpital n'est toutefois qu'un élément intégré à un réseau de parties prenantes (autorités publiques, acteurs politiques ou organisations non gouvernementales selon le contexte, patients, collaborateurs, investisseurs privés et publics, ...) qui interagissent continuellement avec lui : s'ils lui apportent les ressources financières, organisationnelles, humaines, techniques, informationelles dont il a besoin pour son fonctionnement quotidien, ils ont aussi des attentes fortes en terme de performance technique (essentiellement exprimée sous la forme d'une performance sanitaire et de qualité des soins), organisationnelle (essentiellement exprimée en termes de gestion optimale de la rareté des ressources) et financière (essentiellement estimée en termes de respect de contraintes budgétaires ou de maximisation de la valeur actionnariale, selon les contextes). Certaines de ces attentes sont communes à toutes les parties prenantes, (notamment les contraintes techniques) alors que d'autres sont spécifiques à certains groupes de parties prenantes (notamment les contraintes organisationnelles ou financières). 
En conséquence, il apparaît vite erroné de réduire la mission de l'hôpital à la seule et unique mission de prestations d'activités techniques médicales et sa capacité à rencontrer ses obligations à l'égard de ses principales parties prenantes est à considérer comme un prérequis essentiel à la survie économique d'une institution hospitalière et à sa pérennisation.

Simultanément, l'hôpital opère plus que jamais dans un environnement caractérisé par un haut niveau croissant d'incertitude et donc générateur de risques, qui rend encore plus délicate l'atteinte récurrente des buts et objectifs désirés. Cette incertitude trouve son origine notamment dans une augmentation continue de la demande (et donc du besoin en ressources) adressée au monde hospitalier: la croissance continue de la population mondiale, les phénomènes à présent généralisés de viellissement des populations locales, le développement technologique continu et la diffusion de plus en plus rapide des avancées scientifiques, l'automatisation et la numérisation croissante de nombre de tâches et de procesus opérationnels sont autant de facteurs micro- et macro-économiques qui accroissent la probabilité qu'un événement non prévu (inconnu ou non planifié) survienne et l'impact potentiel de cet événement en termes de conséquences néfastes ou de consommation excessive et injustifiée de ressources rares. (McKee, Healy, 2002).

De ce fait, la nécessité d'une gestion préventive, globale et intégrée de l'ensemble des risques techniques, organisationnels et économiques auxquels l'institution hospitalière est confrontée s'impose concommitamment à l'obligation d'efficacité et d'efficience imposée par ailleurs à l'hôpital.

\section{Les caractéristiques managériales de l'institution hospitalière}

Au plan managérial et à l'instar d'une entreprise industrielle, l'hôpital actuel présente dès lors les caractéristiques suivantes, quel que soit son statut ou sa localisation : 
- Dans une vision de l'organisation fondée sur la théorie des ressources (Wernerfelt, 1986), il est constitué d'un ensemble de ressources rares et limitées, de nature humaines, techniques, financières et immatérielles (savoirs et connaissances), dont il doit justifier d'une utilisation efficiente en regard des attentes diverses et parfois contradictoires de ses différentes parties prenantes (patients, collaborateurs, autorités de financement, autorités de contrôle) s'il veut remplir le rôle de création de valeur sociétale naturellement dévolu à toute organisation.

- En terme managérial, son défi quotidien réside dès lors dans la réalisation d'une triple mission :

- Gérer de manière coordonnée des flux multiples de natures diverses (flux des patients physiques, objets des soins ; flux des médicaments et des traitements, nécessaires à la fluidité du flux des patients ; flux d'information, traduction virtuelle des patients physiques nécessaire lui aussi à la fluidité du flux des patients) dans un souci permanent d'efficience multiple : garantir le bon soin au bon patient au bon moment avec le niveau l'information suffisant, les ressources humaines et médicamenteuses et l'infrastructure adéquates, le tout, au moindre coût.

- Coordonner en permanence l'ensemble des ressources humaines, matérielles et financières de l'organisation, et surtout motiver la principale ressource critique, l'ensemble des collaborateurs (corps médical, corps infirmier, corps technicien de support, corps de support à la gestion), en résolvant de manière continue les défis quotidiens d'une bonne communication interpersonnelle (dans et entre les services), d'une motivation individuelle alignée sur les besoins et les attentes de l'organisation et d'une parfaite coordination avec les attentes du patient. 
- Et enfin, déployer au quotidien une stratégie claire, et efficace (donc qui débouche sur les résultats attendus), cohérente en regard des attentes et des objectifs des parties prenantes de l'organisation et des opportunités offertes par son environnement concurrentiel et technologique (dans une logique de partenariat actif). La réalisation de cette troisième mission quotidienne suppose l'existence d'une vision et d'une mission stratégique claire pour l'organisation, par la conception d'un plan stratégique précis avec des objectifs clairs échelonnés dans le temps, par le déploiement d'un système d'information efficient pour supporter la mise en œuvre effective de la stratégie et par une évaluation régulière de sa bonne réalisation.

L'objet qui est au cœur du métier de l'hôpital, le patient, présente toutefois un certain nombre de caractéristiques qui le rendent spécifique : il s'agit d'un objet vivant, amené (via les urgences ou le réseau des prescripteurs) vers l'hôpital de manière non ou peu contrôlée et difficile à réguler ou à anticiper avec précision, avec des besoins (des pathologies) tous différents et donc avec des attentes non uniformes (accueil, qualité de soins, réintégration au domicile et dans la vie courante) et des réactions non uniformes et difficiles à prévoir avec un haut degré de certitude (réactions liées aux traitements et réactions psychologiques).

De ce fait, l'hôpital peut être assimilé à une entreprise industrielle à haut niveau de sécurité attendu : à l'instar de ce type d'industrie (fréquent dans les métiers du nucléaire, de la chimie, de l'agro-alimentaire, du spatial ou de la mécanique de précision, par exemple), il va devoir combiner en permanence des ressources humaines et techniques généralement de pointe et hyper-qualifiées et coordonner au quotidien des ressources toujours trop rares en fonction d'un flux de patients souvent difficile à anticiper.

L'hôpital se trouve ainsi confronté à un défi additionnel : gérer en permanence dans l'urgence des flux potentiellement non prévisibles ou le plus généralement peu prévisibles avec un haut 
niveau de sécurité attendu, créant ainsi un sentiment diffus d'urgence quotidienne permanente.

En conséquence, il va avoir recours naturellement aux techniques de gestion industrielle traditionnellement utilisées dans les environnements à haut niveau de sécurité attendu ("lean management" pour permettre la flexibilité, management par la qualité pour permettre l'efficience organisationnelle, structuration selon le modèle industriel de la "Chaîne de Valeur"...), pour répondre adéquatement aux contraintes d'efficience économique et sociétale, de flexibilité et de sécurité imposées par ses multiples parties prenantes.

Pour permettre l'utilisation efficace de ces techniques de gestion traditionnelles, la mise en place d'un système intégré de contrôle de gestion organisationnelle s'impose vite, complémentaire du système de contrôle de gestion budgétaire ou comptable imposé par le respect des contraintes financières imposées par les parties prenantes de l'hôpital.

Ce système de contrôle de gestion organisationnel intégré comporte alors un ensemble cohérent et structuré de règles, de procédures, de mécanismes et d'outils qui vont permettre à l'hôpital d'agir au niveau de ses acteurs de terrain (corps médical, infirmier, technique, paramédical, managérial) et des sous-ensembles organisationnels qui les regroupent (unités de soins, services, départements, mais aussi corps infirmier, corps médical, corps technique, ...) pour y développer une culture de risque, une culture de sécurité et une culture de performance qui vont ensemble permettre à l'institution hospitalière d'atteindre ses objectifs de manière récurrente tout en générant un niveau de risque économique, sociétal et financier acceptable par les parties prenantes de l'hôpital. 


\section{L'intérêt du modèle des leviers de contrôle de Simons en milieu}

\section{hospitalier}

La mise en œuvre d'un système intégré de contrôle de gestion organisationnelle du risque en milieu hospitalier peut s'appuyer utilement sur le modèle des «leviers de contrôle » proposé par Simons en 1995, utilement adapté aux spécificités et au contexte du monde hospitalier.

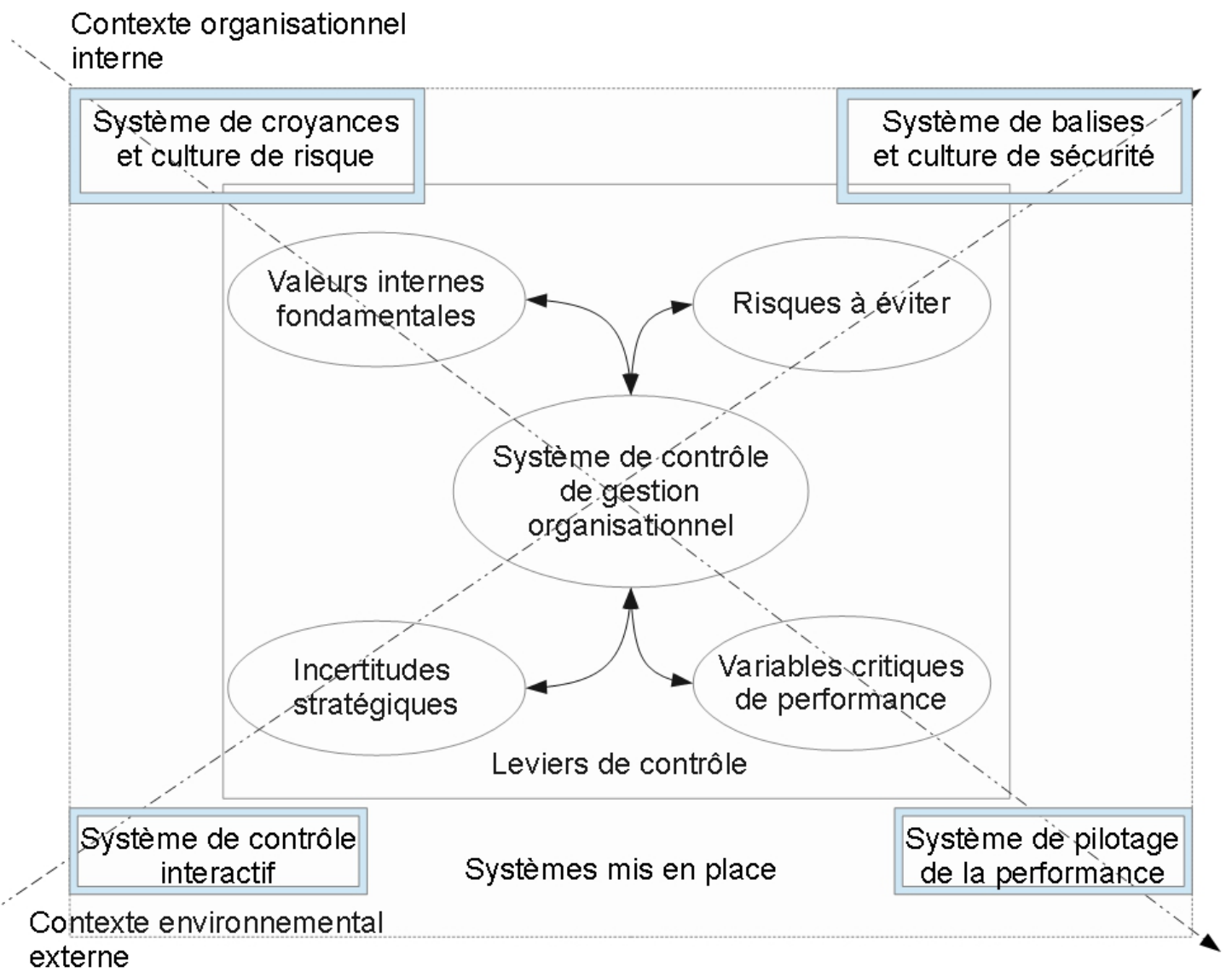

Figure 1: Une adaptation du modèle des leviers de contrôle de Simons en milieu hospitalier (adapté de Simons, 1995).

Au départ du travail liminaire de Simons (1995) et de son questionnement conceptuel (Tessier, Otler, 2012) et après intégration des quelques études déjà consacrées à l'intégration du modèle de Simons au sein des outils de gestion du monde hospitalier (Lartigau, Nobre, 2011), nous pouvons postuler que la stratégie de l'hôpital et le système de contrôle de gestion organisationnelle du risque qui va en permettre la mise en œuvre opérationnelle reposent sur 
quatre leviers de contrôle complémentaires dont il faut assurer à la fois l'équilibre et l'interaction continue :

- Il y a d'une part les leviers imposés par le contexte environnemental externe de l'hôpital, venant donc de ses parties prenantes externes (Pouvoirs Publics, fournisseurs, partenaires scientifiques et financiers, ...), : il s'agit en l'occurrence :

- De la nature des incertitudes stratégiques qui pèsent sur l'hôpital, essentiellement en termes de financement public, de modèle concurrentiel souhaité et d'offre de soins souhaitée et réalisable.

- De la nature des risques opérationnels (risques médicaux, para-médicaux et administratifs) et des risques stratégiques (risques financiers, juridiques, légaux, environnementaux, sociétaux) auxquels l'hôpital se trouve confronté, risques à gérer de manière proactive pour en réduire les effets potentiels.

- Il y a d'autre part les leviers rendus possibles par le contexte organisationnel interne de l'hôpital, qui trouvent leur origine dans les valeurs fondamentales présentes au sein de l'organisation et dans le degré de précision du système d'information, de contrôle et de prise de décision mis en œuvre au sein de l'hôpital (Van Caillie, 2005) : il s'agit en l'occurrence :

- Des valeurs internes fondamentales (qualité technique et médicale des soins, souci du bien-être du patient, attitude de respect, mécanisme de confiance interpersonnelle, souci de conformité, volonté d'autonomie...) qui sont véhiculées au quotidien au sein de l'ensemble des multiples unités organisationnelles présentes au sein de l'hôpital.

- Des variables critiques de performance qui émergent de l'ensemble des trois autres leviers de contrôle interagissant de manière permamente et dont le suivi et le 
pilotage sont rendus effectivement possibles par la précision du système d'information mis en œuvre au sein de l'institution hospitalière.

L'intégration de ces quatre leviers débouche à son tour in fine sur la mise en place de quatre systèmes complémentaires, ensemble cohérent et structuré d'outils, de pratiques, de règles et de mécanismes qui, conjointement, vont constituer le système de contrôle de gestion organisationnelle du risque de l'hôpital :

- Le système de croyances et la culture de risque qui en découle directement, qui vont se fonder sur les valeurs internes fondamentales véhiculées au sein de l'hôpital et les traduire en un système cohérent et structuré de croyances et une culture de risque intégrative de ces croyances, de manière à créer un cadre de référence organisationnel qui va permettre la performance organisationnelle de l'hôpital.

- Le système de balises et la culture de sécurité qui en découle directement, qui vont être construit en fonction des multiples risques opérationnels et stratégiques qui surviennent quotidiennement dans la vie de l'hôpital (risque médical, risque médicamenteux, risque d'approvisionnement, risque technique, mais aussi risque de bien-être, risque de financement et risque de réputation) et des exigences ou contraintes de conformité imposées par les parties prenantes de l'institution hospitalière (normes de qualité, de sécurité, de financement, de niveau de bien-être, $\ldots)$.

- Le système de contrôle interactif, qui va permettre d'ajuster régulièrement le système de contrôle de gestion organisationnelle du risque mis en place à l'évolution des incertitudes stratégiques de l'hôpital au fur et à mesure que celles-ci se lèvent, de manière à rendre ce système de contrôle de gestion organisationnelle adaptatif et autoévolutif. 
- Enfin, le système de pilotage de la performance hospitalière, fondé sur l'intégration cohérente d'une série de tableaux de bord opérationnels (focalisés sur les activités et les processus mis en œuvre au sein des multiples unités organisationnelles présentes au sein de la structure hospitalière) et stratégiques (focalisés sur la mesure du niveau de risque stratégique pesant sur le financement, la qualité de soins et de prestations et la réputation de l'hôpital notamment) sur base d'un système d'information adapté aux différents circuits (circuit du patient, circuit du médicament, circuit administratif, circuit de financement) qui permettent de refléter la réalité du fonctionnement quotidien de l'hôpital.

Dans cette perspective, le système de contrôle de gestion organisationnelle du risque opérationnel et stratégique qui pèse quotidiennement sur l'hôpital devient un système interactif au sein duquel les contrôles humains, techniques et culturels (Merchant, Van der Stede, 2014) mis en œuvre au sein de l'hôpital s'ajustent automatiquement et rapidement à l'évolution de la nature et de l'ampleur des risques qui pèsent sur lui au fur et à mesure que se lèvent les incertitudes stratégiques auxquelles il est confronté, système dont le suivi et le pilotage est rendu possible par l'usage de tableaux de bord stratégiques et opérationnels coordonnés et focalisés sur les variables critiques de performance organisationnelle propres à l'hôpital au sein duquel ce système de contrôle de gestion organisationnelle est appliqué.

Quant aux pratiques managériales hospitalières, elles se focalisent alors essentiellement sur la gestion coordonnée des quatre grands flux interconnectés sur lesquels se cristallise le risque hospitalier, à savoir le flux de patients, le flux technique (soins, médicaments et ressources techniques essentiellement), le flux financier et le flux d'information, de manière à éviter tout décalage intempestif entre ces différents flux (tout décalage induisant un accroissement du niveau du risque sous-jacent). 


\section{Conclusion}

L'analyse développée au long de la présente contribution montre comment un outil de contrôle de gestion organisationnel tel que le modèle des leviers de contrôle de Simons permet, en contexte hospitalier, de construire un ensemble cohérent et structuré de systèmes (système de croyances et culture de risque résultante, système de balises et culture de sécurité résultante, système de contrôle interactif et système de pilotage de la performance) fondé sur les leviers de contrôle propres à l'hôpital (ses valeurs fondamentales, ses incertitudes stratégiques, ses risques à éviter et son ensemble propre de variables critiques de performance qui en résulte) de manière à construire effectivement un système intégré de contrôle de gestion organisationnelle du risque hospitalier.

\section{Bibliographie}

Lartigau, J., Nobre, T. (2011), Une nouvelle grille d'analyse pour le contrôle de gestion hospitalier: Le contrôle intégré de Simons. Comptabilités, Economie et Société. Montpellier, France.

McKee, M., Healy, J. (Eds.) (2002), Hospitals in a changing Europe. Open University Press, Buckingham, Philadelphia.

Merchant K., Van der Stede W. (2011), Management Control Systems - Performance Measurement, Evaluation and Incentives. 3 ème édition. New-York : Prentice-Hall

Milgrom P., Roberts J. (1997) Economie, organisation et management. Collection « Ouvertures économiques - Balises ». Bruxelles : De Boeck Université

Simons, R. (1995), Levers of control: how managers use innovative control systems to drive strategic renewal. Harvard Business School Press, Boston.

Tessier, S., Otley, D. (2012), A conceptual development of Simons' levers of control framework, Management Accounting Research, Vol. 23 No. 3, pp. 171-185.

Van Caillie D. (2005), Principes de comptabilité analytique et de comptabilité de gestion. 2 ème édition. Liège : Presses Universitaires de Liège

Wernerfelt B. (1984), A resource-based view of the firm, Strategic Management Journal vol. 5 : $171-180$ 
Widener, S. (2007), An empirical analysis of the levers of control framework, Accounting, Organizations and Society, Vol. 32, pp. 757-788.

WHO World Health Organization (1963), Expert committee on health statistics. Eight report. Technical Report Series №261. Geneva.

WHO World Health Organization (2013a). Research for Universal Health Coverage, available at: http://apps.who.int/iris/bitstream/10665/85761/2/978924069

0837_eng.pdf (accessed 13 October 2013).

WHO World Health Organization (2013b), World Health Statistics, available at: http://www.who.int/gho/publications/world_health_statistics/2013/en/(accessed 13 October 2013). 